\title{
A NOTE ON WEAKLY QUASI CONTINUOUS FUNCTIONS
}

\author{
JIN HAN PARK \\ Department of Natural Sciences \\ Pusan National University of Technology \\ Yongdang-dong Nam-ku, Pusan, 608-739, KOREA \\ HOE YOUNG HA \\ Department of Mathematics \\ Inje University \\ Obang-dong, Kimhae, 621-749, KOREA
}

(Received September 27, 1994 and in revised form February 25, 1995)

ABSTRACT. The notion of weakly quasi continuous functions introduced by Popa and Stan [1] In this paper, the authors obtain the further properties of such functions and introduce weak* quasi continuity which is weaker than semi continuity [2] but independent of weak quasi continuity.

KEY WORDS AND PHRASES. Weakly continuous, semi continuous, weakly quasi continuous, weakly* quasi continuous.

1991 AMS SUBJECT CLASSIFICATION CODE. 54C08.

\section{INTRODUCTION}

As weak forms of continuity in topological spaces, semi continuity, weak continuity [3], quasi continuity [4] and almost continuity in the sense of Husain [5] are well known. Neubrunnová [6] showed that semi continuity is equivalent to quasi continuity. Also, Noiri [7] showed that semi continuity, weak continuity and almost continuity are respectively independent. In 1973, Popa and Stan [1] introduced weak quasi continuity which is implied by both weak $\alpha$-continuity [8] and semi continuity. It is shown in [7] that weak quasi continuity is equivalent to weak semi continuity due to Arya and Bhamini [9]. Recently, Noiri in $[7,8]$ investigated fundamental properties of weakly quasi continuous functions and compared the interrelation among weak quasi continuity, weak $\alpha$-continuity, semi continuity and almost continuity.

The purpose of this paper is to obtain some characterizations of weakly quasi continuous functions and investigate the relationships between such functions and some separation axioms. We also introduce weak* quasi continuity which is weaker than semi continuity but independent of weak quasi continuity.

\section{PRELIMINARIES}

Throughout the present paper, spaces always mean topological spaces and $f: X \rightarrow Y$ denotes a single valued function of a space $X$ into a space $Y$. Let $X$ be a space and $A$ a subset of $X$. We denote the closure of $A$ and the interior of $A$ by $\mathrm{Cl}(A)$ and $\operatorname{Int}(A)$, respectively. A subset $A$ is said to be semiopen [2] (resp. preopen [10], $\alpha$-open [11]) if $A \subset \mathrm{Cl}(\operatorname{Int}(A)$ ) (resp. $A \subset \operatorname{Int}(\mathrm{Cl}(A)$ ), $A \subset \operatorname{Int}(\mathrm{Cl}(\operatorname{Int}(A))))$. We denote the family of semiopen (resp. preopen, $\alpha$-open) sets of $X$ by $S O(X)$ (resp. $P O(X), \alpha(X)$ ). It is shown that $\alpha(X)=S O(X) \cap P O(X)$ [12]. The complement of a 
semiopen set is said to be semiclosed The intersection of all semiclosed sets containing $A$ is called the semi-closure [13] of $A$ and is denoted by $s-\mathrm{Cl}(A)$. The semi-interior [13] of $A$, denoted by $s-\operatorname{Int}(A)$, is defined by the union of all semiopen sets contained in $A$. A subset $A$ of $X$ is said to be regular open (resp regular closed) [14] if $A=\operatorname{Int}(\mathrm{Cl}(A))$ (resp $A=\mathrm{Cl}(\operatorname{Int}(A))$ ). A point $x \in X$ is in the $\theta$-closure of $A$ [15], denoted by $\mathrm{Cl}_{\theta}(A)$, if $A \cap \mathrm{Cl}(U) \neq \emptyset$ for each open set $U$ containing $x$. A subset $A$ is called $\theta$-closed if $\mathrm{Cl}_{\theta}(A)=A$

DEFINITION A. A function $f: X \rightarrow Y$ is said to be

(a) semi continuous [2] (briefly, $\mathrm{s} \mathrm{c} \mathrm{)} \mathrm{if} f^{-1}(V) \in S O(X)$ for each open set $V$ of $Y$;

(b) almost continuous [5] if for each $x \in X$ and each open set $V$ containing $f(x), \mathrm{Cl}\left(f^{-1}(V)\right)$ is a neighborhood of $x$;

(c) weakly continuous [3] (resp. $\theta$-continuous [16]) if for each $x \in X$ and each open set $V$ containing $f(x)$, there exists an open set $U$ containing $x$ such that $f(U) \subset \mathrm{Cl}(V)$ (resp $f(\mathrm{Cl}(U)) \subset \mathrm{Cl}(V))$;

(d) weakly $\alpha$-continuous [8] (briefly, w. $\alpha . c$.) if for each $x \in X$ and each open set $V$ containing $f(x)$, there exists a $U \in \alpha(X)$ containing $x$ such that $f(U) \subset \mathrm{Cl}(V)$.

\section{WEAKLY QUASI CONTINUOUS FUNCTIONS \\ DEFINITION 3.1. A function $f: X \rightarrow Y$ is said to be}

(a) weakly quasi continuous [1] (briefly, w.q.c.) if for each $x \in X$, each open set $G$ containing $x$ and each open set $V$ containing $f(x)$, there exists an open set $U$ of $X$ such that $\emptyset \neq U \subset G$ and $f(U) \subset \mathrm{Cl}(V)$;

(b) weakly semi-continuous [9] (briefly, w.s.c.) if for each $x \in X$ and each open set $V$ containing $f(x)$, there exists a $U \in S O(X)$ containing $x$ such that $f(U) \subset \mathrm{Cl}(V)$.

Noiri showed in [7, Theorem 4.1] that a function $f: X \rightarrow Y$ is w.q.c. if and only if for each $x \in X$ and each open set $V$ containing $f(x)$, there exists a $U \in S O(X)$ containing $x$ such that $f(U) \subset \mathrm{Cl}(V)$. Hence we know that w.q.c. and w.s.c. are equivalent concepts.

The following is shown in [7, Theorem 4.2, 4.3] and [8, Lemma 5.3].

THEOREM 3.2. For a function $f: X \rightarrow Y$, the following are equivalent:

(a) fis w.q.c.

(b) For each subset $B$ of $Y, s-C l\left(f^{-1}(\operatorname{Int}(C l(B)))\right) \subset f^{-1}(C l(B))$.

(c) For each regular closed set $F$ of $Y, s-C l\left(f^{-1}(\operatorname{Int}(F))\right) \subset f^{-1}(F)$.

(d) For each open set $B$ of $Y, s-C l\left(f^{-1}(B)\right) \subset f^{-1}(C l(B))$.

(e) For each open set $B$ of $Y, f^{-1}(B) \subset s-\operatorname{Int}\left(f^{-1}(C l(B))\right)$.

(f) For each regular closed set $B$ of $Y, f^{-1}(B) \in S O(X)$.

(g) For each open set $B$ of $Y, f^{-1}(B) \subset C l\left(\operatorname{Int}\left(f^{-1}(C l(B))\right)\right)$.

THEOREM 3.3. For a function $f: X \rightarrow Y$, the following are equivalent:

(a) fis w.q.c.

(b) For each subset $B$ of $Y, s-C l\left(f^{-1}(B)\right) \subset f^{-1}\left(C l_{\theta}(B)\right)$.

(c) For each subset $A$ of $X, f(s-C l(A)) \subset C l_{\theta}(f(A))$.

(d) For each subset $A$ of $X, f(\operatorname{Int}(C l(A))) \subset C l_{\theta}(f(A))$.

(e) For each subset $B$ of $Y, \operatorname{Int}\left(C l\left(f^{-1}(B)\right)\right) \subset f^{-1}\left(C l_{\theta}(B)\right)$.

(f) For each open set $B$ of $Y, \operatorname{Int}\left(C l\left(f^{-1}(B)\right)\right) \subset f^{-1}(C l(B))$.

PROOF. It follows immediately from Theorem 3.2 and [17, Theorem 1.5].

THEOREM 3.4. A function $f: X \rightarrow Y$ is a w.q.c. if and only if for each subset $B$ of $Y$, ${ }_{s-C l}\left(f^{-1}\left(\operatorname{Int}\left(C l_{\theta}(B)\right)\right)\right) \subset f^{-1}\left(C l_{\theta}(B)\right)$.

PROOF. Necessity. Let $B$ be a subset of $Y$. Assume that $x \notin f^{-1}\left(\mathrm{Cl}_{\theta}(B)\right)$. Then $f(x) \notin \mathrm{Cl}_{\theta}(B)$ and hence there exists an open set $W$ containing $f(x)$ such that $B \cap \mathrm{Cl}(W)=\emptyset$. This 
implies that $\mathrm{Cl}_{\theta}(B) \cap W=\emptyset$ and so $W \subset Y-\mathrm{Cl}_{\theta}(B)$, i e $\mathrm{Cl}(W) \subset \mathrm{Cl}\left(Y-\mathrm{Cl}_{\theta}(B)\right)$ Since $f$ is w q c, there exists a $U \in S O(X)$ containing $x$ such that $f(U) \subset \mathrm{Cl}(W) \subset \mathrm{Cl}\left(Y-\mathrm{Cl}_{\theta}(B)\right)$ This implies that $U \cap f^{1}\left(\operatorname{Int}\left(\mathrm{Cl}_{\theta}(B)\right)\right)=\emptyset$ and hence $x \notin s-\mathrm{Cl}\left(f^{1}\left(\operatorname{Int}\left(\mathrm{Cl}_{\theta}(B)\right)\right)\right) \quad$ Therefore, $s-\mathrm{Cl}\left(f^{1}\left(\operatorname{Int}\left(\mathrm{Cl}_{\theta}(B)\right)\right)\right) \subset f^{1}\left(\mathrm{Cl}_{\theta}(B)\right)$

Sufficiency Let $B$ be an open set of $Y$ Then clearly $\mathrm{Cl}(B)=\mathrm{Cl}_{\theta}(B)$ By hypothesis, we have $s-\mathrm{Cl}\left(f^{1}(\operatorname{Int}(\mathrm{Cl}(B)))\right)=s-\mathrm{Cl}\left(f^{-1}\left(\operatorname{Int}\left(\mathrm{Cl}_{\theta}(B)\right)\right)\right) \subset f^{1}\left(\mathrm{Cl}_{\theta}(B)\right)=f^{1}(\mathrm{Cl}(B)) \quad$ Hence, by Theorem $32, f$ is w q c

The composition of two $w \mathrm{q} \mathrm{c}$ functions may fail to be w q c [7] But Noiri showed in [7, Theorem 6 1 6] that under certain conditions the composition of two functions is $\mathrm{w} \mathrm{q} \mathrm{c}$

THEOREM 3.5. Let $f: X \rightarrow Y$ and $g: Y \rightarrow Z$ be functions.

(a) If $f$ is w.q.c. and $g$ is $\theta$-contınuous, then $g \circ f$ is w.q.c.

(b) If $f$ is s.c. and $g$ is weakly continuous, then $g \circ f$ is w.q.c.

PROOF. (a) Let $x \in X$ and $W$ be an open set of $Z$ containing $g(f(x))$ Since $g$ is $\theta$-continuous, there exists an open set $V$ of $Y$ containing $f(x)$ such that $g(\mathrm{Cl}(V)) \subset \mathrm{Cl}(W)$ Since $f$ is w q c, there exists a $U \in S O(X)$ containing $x$ such that $f(U) \subset \mathrm{Cl}(V)$ Hence $g(f(U)) \subset g(\mathrm{Cl}(V)) \subset \mathrm{Cl}(W)$

(b) The proof is easy and hence omitted

COROLLARY 3.6 (Noiri [7]) If $f: X \rightarrow Y$ is w.q.c. and $g: Y \rightarrow Z$ is contmuous, then $g \circ f$ is w.q.c.

LEMMA 3.7 (Noiri and Ahmad [18]) Let $A$ and $B$ be subsets of $X$. If $A \in P O(X)$ and $B \in S O(X)$, then $A \cap B \in S O(X)$.

THEOREM 3.8. If $f: X \rightarrow Y$ is w.q.c. and $A \in P O(X)$, then the restriction $\left.f\right|_{A}: A \rightarrow Y$ is w.q.c.

PROOF. Let $x \in A$ and $V$ be an open set of $Y$ containing $f(x)$ Since $f$ is w q c, there exists a $U \in S O(X)$ containing $x$ such that $f(U) \subset \mathrm{Cl}(V)$ Since $A \in P O(X)$, by Lemma 37 $x \in A \cap U \in S O(X)$ and $\left(\left.f\right|_{A}\right)(A \cap U)=f(A \cap U) \subset f(U) \subset \mathrm{Cl}(V)$ Hence $\left.f\right|_{A}$ is w q c

COROLLARY 3.9 (Noiri [7]) If $f: X \rightarrow Y$ is w.q.c. and $A$ is open in $X$, then the restriction $\left.f\right|_{A}: A \rightarrow Y$ is w.q.c.

COROLLARY 3.10 (Arya and Bhamini [9]) If $f: X \rightarrow Y$ is w.q.c. and $A \in \alpha(X)$, then the restriction $\left.f\right|_{A}: A \rightarrow Y$ is w.q.c.

Sufficient condition for a function to be $\mathrm{w} \mathrm{q} \mathrm{c}$, when it is given to be so in some subspace, is given in the following

THEOREM 3.11. Let $f: X \rightarrow Y$ be a function and $\left\{A_{2} \mid i \in I\right\}$ be a cover of $X$ such that $A_{\imath} \in S O(X)$ for each $i \in I$. If $\left.f\right|_{A_{2}}: A_{\imath} \rightarrow Y$ is w.q.c. for each $i \in I$, then $f$ is w.q.c.

PROOF. Let $V$ be a regular closed set of $Y$. Then $\left(\left.f\right|_{A_{1}}\right)^{-1}(V) \in S O\left(A_{2}\right)$ Since $A_{2} \in S O(X)$, by Theorem 24 of [19], $\left(\left.f\right|_{A_{1}}\right)^{-1}(V) \in S O(X)$ for each $i \in I \quad$ But $f^{-1}(V)=\cup_{\imath \in I}\left(\left(\left.f\right|_{A_{1}}\right)^{-1}(V)\right)$ Then $f^{-1}(V) \in S O(X)$ because the union of semiopen sets is semiopen [2] Hence, by Theorem $32 f$ is $\mathrm{w} \mathrm{q} \mathrm{c}$

COROLLARY 3.12. Let $f: X \rightarrow Y$ be a function and $\left\{A_{2} \mid i \in I\right\}$ be a cover of $X$ such that $A_{\imath} \in \alpha(X)$ for each $i \in I$. If $\left.f\right|_{A_{2}}: A_{\imath} \rightarrow Y$ is w.q.c. for each $i \in I$, then $f$ is w.q.c.

COROLLARY 3.13. Let $f: X \rightarrow Y$ be a function and $\left\{A_{2} \mid i \in I\right\}$ be a cover of $X$ such that $A_{2}$ is open in $X$ for each $i \in I$. If $\left.f\right|_{A_{2}}: A_{\imath} \rightarrow Y$ is w.q.c. for each $i \in I$, then $f$ is w.q.c.

DEFINITION 3.14. Let $A$ be a subset of $X$ A function $f: X \rightarrow A$ is called a w q c retraction if $f$ is w q c and $\left.f\right|_{A}$ is the identity function on $A$

THEOREM 3.15. Let $A$ be a subset of $X$ and $f: X \rightarrow A$ be a w.q.c. retraction. If $X$ is $T_{2}$, then A is semiclosed in $X$. 
PROOF. Suppose that $A$ is not semiclosed Then there exists a $x \in X$ such that $x \in s$ $\mathrm{Cl}(A)-A$ Since $f$ is a w q c retraction, $f(x) \neq x$ By the $T_{2}$ property of $X$, there exist disjoint open sets $U$ and $V$ such that $x \in U$ and $f(x) \in V$ which implies $U \cap \mathrm{Cl}(V)=\emptyset$. Let $W \in S O(X)$ containing $x$. Then $U \cap W \in S O(X)$ and hence $(U \cap W) \cap A \neq \emptyset$ because $x \in s-\operatorname{Cl}(A)$. Let $y \in(U \cap W) \cap A$. Since $y \in A$, we have $f(y)=y \in U \cap W \cap A \subset U$ and hence $f(y) \notin \mathrm{Cl}(V)$ This implies that $f(W) \not \subset \mathrm{Cl}(V)$ because $y \in W$. This is contrary to the fact that $f$ is w q c Hence $A$ is semiclosed in $X$.

In [8], Noiri showed that if $Y$ is $T_{2}, f_{1}: X \rightarrow Y$ is s c., $f_{2}: X \rightarrow Y$ is w $\alpha . c$ and $f_{1}=f_{2}$ on a dense subset of $X$, then $f_{1}=f_{2}$ on $X$ Similarly, we have

THEOREM 3.16. Let $Y$ be $T_{2}$ and $f_{1}: X \rightarrow Y$ be almost continuous. If $f_{2}: X \rightarrow Y$ is w.q.c. and if $f_{1}=f_{2}$ on a dense subset $D$ of $X$, then $f_{1}=f_{2}$ on $X$.

PROOF. Similar to the proof of [8, Theorem 4 10] by using Lemma 3.7.

THEOREM 3.17. Let $Y$ be Urysohn and $f_{1}: X \rightarrow Y$ be w.q.c. If $f_{2}: X \rightarrow Y$ is w.a.c. and if $f_{1}=f_{2}$ on a dense subset $D$ of $X$, then $f_{1}=f_{2}$ on $X$.

PROOF. Similar to the proof of [8, Theorem 4.10].

\section{GRAPHS OF FUNCTIONS}

The graph of a function $f: X \rightarrow Y$, denoted by $G(f)$, is the subset $\{(x, f(x)) \mid x \in X\}$ of the product space $X \times Y$. Noiri [20] showed that if $f: X \rightarrow Y$ is weakly continuous and $Y$ is $T_{2}$, then the graph $G(f)$ is closed. Using "w.q.c." and "semiclosed" instead of "weakly continuous" and "closed" respectively, we obtain the following.

THEOREM 4.1. If $f: X \rightarrow Y$ is w.q.c. and $Y$ is $T_{2}$, then for each $(x, y) \notin G(f)$, there exist $U \in S O(X)$ and open set $V$ in $X$ such that $x \in U, y \in V$ and $f(U) \cap \operatorname{Int}(C l(V))=\emptyset$.

PROOF. Let $(x, y) \notin G(f)$. Then $y \neq f(x)$. Since $Y$ is $T_{2}$, there exist disjoint open sets $V$ and $W$ such that $y \in V$ and $f(x) \in W$. This implies that $\operatorname{Int}(\mathrm{Cl}(V)) \cap \mathrm{Cl}((W)=\emptyset$. Since $f$ is w.q.c., there exists $U \in S O(X)$ containing $x$ such that $f(U) \subset \mathrm{Cl}(W)$. Hence $f(U) \cap \operatorname{Int}(\mathrm{Cl}(V))=\emptyset$.

COROLLARY 4.2. If $f: X \rightarrow Y$ is w.q.c. and $Y$ is $T_{2}$, then the graph $G(f)$ is semiclosed.

PROOF. It follows from Theorem 4.1.

THEOREM 4.3. If $f: X \rightarrow Y$ is a w.q.c. and $S$ is $\theta$-closed subset in $X \times Y$, then $p_{1}(S \cap G(f))$ is semiclosed in $X$, where $p_{1}$ is the projection of $X \times Y$ onto $X$.

PROOF. Let $x \in s-\mathrm{Cl}\left(p_{1}(S \cap G(f))\right)$, where $S$ is a $\theta$-closed subset of $X \times Y$.

Let $U$ and $V$ be any open sets of $X$ and $Y$ containing $x$ and $f(x)$, respectively. Since $f$ is w.q.c., by Theorem $3.2 x \in f^{-1}(V) \subset s-\operatorname{Int}\left(f^{-1}(\mathrm{Cl}(V))\right)$. Since $U \cap s-\operatorname{Int}\left(f^{-1}(\mathrm{Cl}(V))\right) \in S O(X)$ containing $x,\left(U \cap s-\operatorname{Int}\left(f^{-1}(\mathrm{Cl}(V))\right)\right) \cap p_{1}(S \cap G(f)) \neq \emptyset$. Let $x_{0} \in\left(U \cap s-\operatorname{Int}\left(f^{-1}(\mathrm{Cl}(V))\right)\right) \cap p_{1}(S \cap G(f))$. This implies that $\left(x_{0}, f\left(x_{0}\right)\right) \in S$ and $f\left(x_{0}\right) \in \mathrm{Cl}(V)$. Therefore, $\phi \neq(U \times \mathrm{Cl}(V)) \cap S \subset$ $\mathrm{Cl}(U \times V) \cap S$ and consequently, $(x, f(x)) \in \mathrm{Cl}_{\theta}(S)$. Since $S$ is $\theta$-closed, $(x, f(x)) \in S \cap G(f)$. Hence $x \in p_{1}(S \cap G(f))$. This shows that $p_{1}(S \cap G(f))$ is semiclosed in $X$.

COROLLARY 4.4. If $f: X \rightarrow Y$ has a $\theta$-closed graph $G(f)$ and $g: X \rightarrow Y$ is w.q.c., then $\{x \in X \mid f(x)=g(x)\}$ is semiclosed.

PROOF. Since $\{x \in X \mid f(x)=g(x)\}=p_{1}(G(f) \cap G(g))$ and $G(f)$ is a $\theta$-closed subset of $X \times Y$, it follows from Theorem 4.3 that $\{x \in X \mid f(x)=g(x)\}$ is semiclosed.

COROLLARY 4.5. If $f: X \rightarrow Y$ is $\theta$-continuous, $g: X \rightarrow Y$ is w.q.c. and $Y$ is Urysohn, then $\{x \in X \mid f(x)=g(x)\}$ is semiclosed.

PROOF. It follows from Theorem 7 of [21] and Corollary 4.4 .

DEFINITION 4.6. Let $f: X \rightarrow Y$ be a function. The graph $G(f)$ is said to be strongly semiclosed if for each $(x, y) \in X \times Y-G(f)$, there exist $U \in S O(X)$ and $V \in S O(Y)$ such that $x \in U, y \in V$ and $(U \times s-\mathrm{Cl}(V)) \cap G(f)=\emptyset$. 
LEMMA 4.7. If $f: X \rightarrow Y$ has a strongly semiclosed graph $G(f)$ if and only if for each $(x, y) \in X \times Y-G(f)$ there exist $U \in S O(X)$ and $V \in S O(Y)$ such that $x \in U, y \in V$ and $f(U) \cap s-\mathrm{Cl}(V)=\emptyset$

PROOF. It follows from Definition 46

THEOREM 4.8. If $f: X \rightarrow Y$ is w.q.c. and $Y$ is Urysohn, then $G(f)$ is strongly semiclosed in $X \times Y$.

PROOF. Since $s-\mathrm{Cl}(U) \subset \mathrm{Cl}(U)$ for each subset $U$ of $X$, it follows Immediately from Lemma 47

\section{WEAK* QUASI CONTINUITY}

DEFINITION 5.1. A function $f \cdot X \rightarrow Y$ is weakly* quasi contınuous (briefly, $\mathrm{w}^{*} \mathrm{q}$ c) if for each open set $V$ of $Y, f^{-1}(\operatorname{Fr}(V))$ is semiclosed in $X$, where $\operatorname{Fr}(V)$ denotes the frontier of $V$

Every s c function is $\mathrm{w}^{*} \mathrm{q} \mathrm{c}$ but the converse is not true as the following Example 52 shows Moreover, Example 52 and 53 show that $\mathrm{w} \mathrm{q} \mathrm{c}$ and $\mathrm{w}^{*} \mathrm{q} \mathrm{c}$ are independent of each other

EXAMPLE 5.2. Let $X=\{a, b, c\}, \tau=\{\phi, X,\{a\}\}$ and $\sigma=\{\phi, X,\{a\},\{b c\}\} \quad$ Let $f(X, \tau) \rightarrow(X, \sigma)$ be the identity function Then $f$ is $\mathrm{w}^{*} \mathrm{q} \mathrm{c}$ However, $f$ is not $\mathrm{s} \mathrm{c}$ and hence not w q c

EXAMPLE 5.3. Let $X=\{a, b, c\}, \tau=\{\phi, X,\{a\}\} \quad$ and $\quad \sigma=\{\phi, X,\{b\}\} \quad$ Let $f:(X, \tau) \rightarrow(X \sigma)$ be the identity function Then $f$ is $\mathrm{w} \mathrm{q} \mathrm{c}$ but $f$ is not $\mathrm{w}^{*} \mathrm{q} \mathrm{c}$

The $w \mathrm{q} c$ functions are not generally $\mathrm{s} \mathrm{c}$ [7] The next two theorems give conditions under which w q c and s c functions are equivalent A space $X$ is said to be extremally disconnected if the closure of each open set is open in $X$

THEOREM 5.4. Let $f: X \rightarrow Y$ be a function and $X$ be extremally disconnected. Then $f$ is s.c. if and only if $f$ is w.q.c. and $w^{*}$.q.c.

PROOF. The necessity is clear

Sufficiency Let $x \in X$ and $V$ be any open set containing $f(x)$ Since $f$ is $\mathbf{w ~ q ~ c ~ , ~ t h e r e ~ e x i s t s ~ a ~}$ $U \in S O(X)$ containing $x$ such that $f(U) \subset \mathrm{Cl}(V)$ But since $f$ is $\mathrm{w}^{*} \mathrm{q}$ c , $f^{-1}(\operatorname{Fr}(V))=f^{-1}(\mathrm{Cl}(V)-V) \quad$ is semiclosed and hence by Proposition of [22] $U-f^{-1}(F r(V)) \in S O(X) \quad$ Further $f(x) \notin F r(V)$ implies $x \notin f^{-1}(\operatorname{Fr}(V)) \quad$ The proof will be complete if we show that $f(x) \in f\left(U-f^{-1}(\operatorname{Fr}(V))\right) \subset V$ Let $y \in U-f^{-1}(\operatorname{Fr}(V))$ Then $f(y) \in \mathrm{Cl}(V)$ But $y \notin f^{-1}(F r(V))$ and so $f(y) \notin F r(V)=\mathrm{Cl}(V)-V$ which implies that $f(y) \in V$

In Theorem 5.4, we cannot drop the assumption that $X$ is extremally disconnected as Example 55 shows

EXAMPLE 5.5. Let $X=\{a, b, c, d\}, \tau=\{\phi, X,\{b\},\{c\},\{b, c\},\{a, b, c\},\{b, c, d\}\}$ and $\sigma=\{\phi, X,\{a\},\{c\},\{a, c\},\{a, b, c\}\} \quad$ Let $f:(X, \tau) \rightarrow(X, \sigma)$ be the identity function Then $f$ is $\mathrm{w} \mathrm{qc}$ and $\mathrm{w}^{*} \mathrm{q} \mathrm{c}$ but not $\mathrm{s} \mathrm{c}$

A space $X$ is said to be rim-compact [14] if each point of $X$ has a base of neighborhoods with compact frontiers

THEOREM 5.6. If $f: X \rightarrow Y$ is w.q.c. with the closed graph $G(f)$ and $Y$ is rim-compact, then f is s.c.

PROOF. Let $x \in X$ and $V$ be any open set containing $f(x)$ Since $Y$ is rim-compact, there exists an open set $W$ of $Y$ such that $f(x) \in W \subset V$ and $F r(W)$ is compact Because $f$ is w q c, there exists a $U \in S O(X)$ containing $x$ such that $F(U) \subset \mathrm{Cl}(W)$ Let $y \in F r(W)$ Since $f(x) \in W$ which is disjoint from $\operatorname{Fr}(W),(x, y) \notin G(f)$ Then since $G(f)$ is closed, there exist open sets $U_{y}$ and $V_{y}$ such that $x \in U_{y}, y \in V_{y}$ and $f\left(U_{y}\right) \cap V_{y}=\emptyset$ The collection $\left\{V_{y} \mid y \in \operatorname{Fr}(W)\right\}$ is an open cover of $\operatorname{Fr}(W)$ Since $\operatorname{Fr}(W)$ is compact, there exist a finite number of points $y_{1}, y_{2}, \ldots, y_{n}$ in $\operatorname{Fr}(W)$ such that $\operatorname{Fr}(W) \subset \cup_{\imath=1}^{n} V_{y_{\imath}}$ Let $U_{0}=U \cap\left(\cup_{\imath=1}^{n} U_{y_{\imath}}\right) \quad$ Then $U_{0} \in S O(X)$ and 


$$
f\left(U_{0}\right) \subset f\left(\cap_{\imath=1}^{n} U_{y_{1}}\right) \subset \cap_{\imath=1}^{n} f\left(U_{y_{1}}\right)
$$

which is disjoint from $\cup_{i=1}^{n} V_{y_{1}}$ and hence disjoint from $\operatorname{Fr}(W)$. Thus $f\left(U_{0}\right) \cap \operatorname{Fr}(W)=\emptyset$. However $f\left(U_{0}\right) \subset f(U) \subset \mathrm{Cl}(W)$ Therefore, $f\left(U_{0}\right) \subset \mathrm{Cl}(W)-F r(W) \subset W$ Hence $f$ is s.c.

\section{REFERENCES}

[1] POPA, V. and STAN, C, On a decomposition of quasi-continuity in topological spaces (Romanian), Stud, Cerc. Math 25 (1973), 41-43.

[2] LEVINE, N., Semi-open sets and semi-continuity in topological spaces, Amer. Math. Monthly 70 (1963), 36-41

[3] LEVINE, N., A decomposition of continuity in topological spaces, Amer. Math. Monthly 68 (1961), 44-46.

[4] MARCUS, S., Sur les fonctions quasi continues au sens de S. Kempisty, Colloq. Math. 8 (1961), 47-53.

[5] HUSAIN, T., Almost continuous mappings, Prace Math. 10 (1966), 1-7.

[6] NEUBRUNNOVÁ, A., On transfinite convergence and generalized continuity, Math. Slovaca 30 (1980), 51-56.

[7] NOIRI, T., Properties of some weak forms of continuity, Internat. J. Math. \& Math. Sci. 10 (1987), 97-111

[8] NOIRI, T., Weakly $\alpha$-continuous functions, Internat. J. Math. \& Math. Sci. 10 (1987), 483-490.

[9] ARYA, S. P. and BHAMINI, M. P., Some weaker forms of semi-continuous functions, Ganita 33 (1982), 124-134.

[10] MASHHOUR, A.S., HASANEIN, I.A. and EL-DEEB, S.N., $\alpha$-continuous and $\alpha$-open mappings, Acta Math. Hung. 41 (1983), 213-218.

[11] NJÅSTAD, O., On some classes of nearly open sets, Pacific J. Math. 15 (1965), 961-970.

[12] NOIRI, T., On $\alpha$-continuous functions, Casopis Pêst. Math. 109 (1984), 118-126.

[13] CROSSLEY, S.G. and HILDEBRAND, S.K., Semi-closure, Texas J. Sci. 22 (1971), 99-112.

[14] WILlaRD, S., General Topology, Addison-Wesley Publishing Company, 1970.

[15] VELIĈKO, N.V., H-closed topological spaces, Amer. Math. Soc. Transl. (2) 78 (1968), 103-118.

[16] FOMIN, S.V., Extensions of topological spaces, Ann. Math. 44 (1943), 471-480.

[17] ANDRIJEVIĆ, P., Semi-preopen sets, Mat. Vesnik 38 (1986), 24-32.

[18] NOIRI, T. and AHMAD, B., A note on semi-open functions, Math. Sem. Notes Kobe Univ. 10 (1982), 437-441.

[19] MAHESHWARI, S.N. and PRASAD, R., Some new separation axioms, Annales de la Soc. Sci. Bruxelles T-89 III (1975), 395-402.

[20] NOIRI, T., Weak continuity and closed graph, Casopis Pêst. Mat. 101 (1976), 379-382.

[21] NOIRI, T., Properties of $\theta$-continuous functions, Att. Accad. Naz. Lincei Rend. Cl. Sci. Fis. Mat. Natur. Serie 8, 58, Fasc. 6 (1975), 887-891.

[22] JANKOVIĆ, D.S., A note on semihomeomorphism between semi- $T_{D}$ spaces, Math. Vesnik 34 (1982), 379-381. 


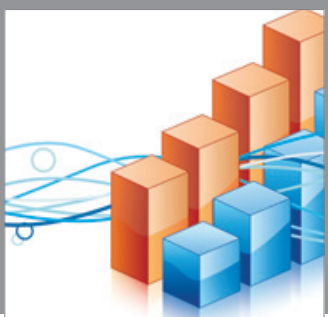

Advances in

Operations Research

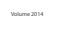

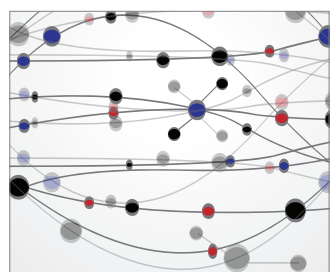

\section{The Scientific} World Journal


International Journal of

Mathematics and

Mathematical

Sciences
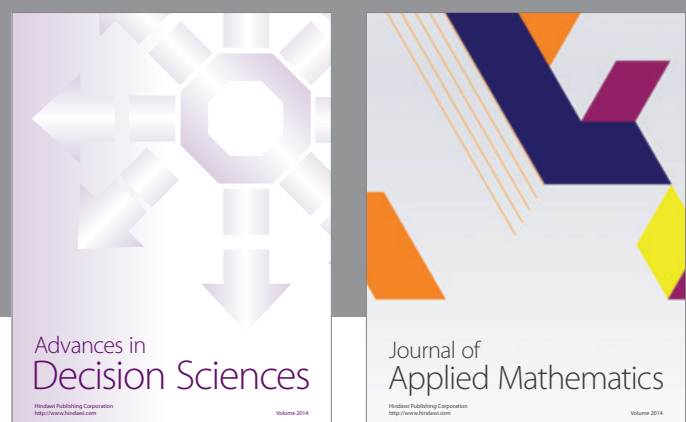

Journal of

Applied Mathematics


Submit your manuscripts at http://www.hindawi.com
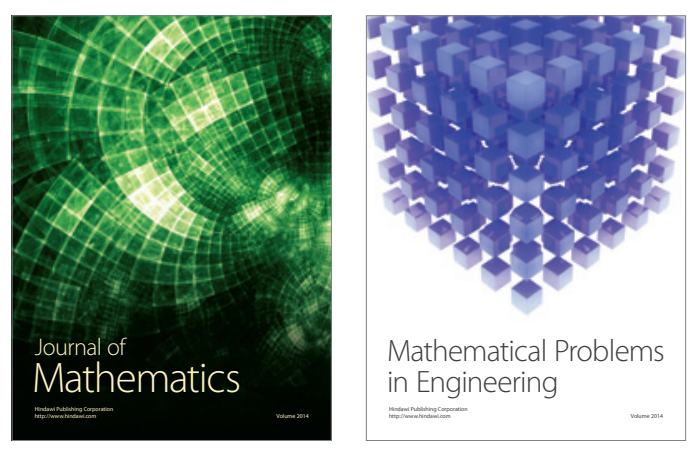

Mathematical Problems in Engineering
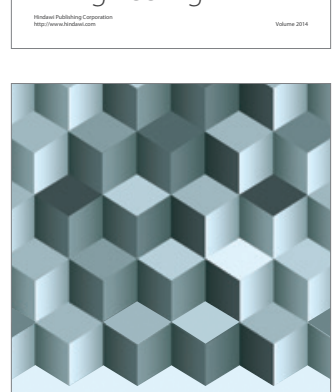

Journal of

Function Spaces
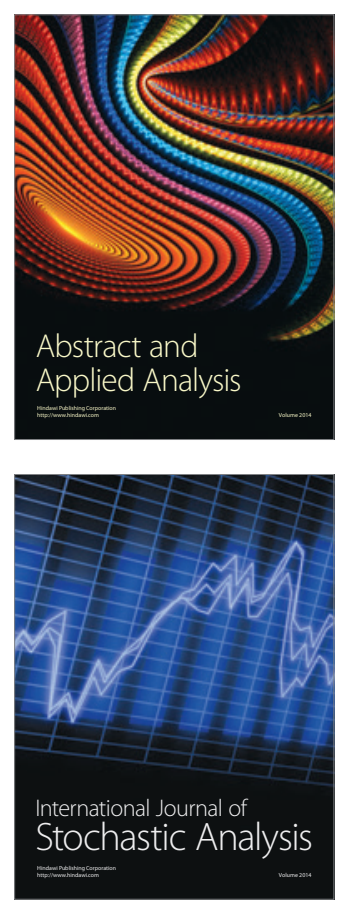

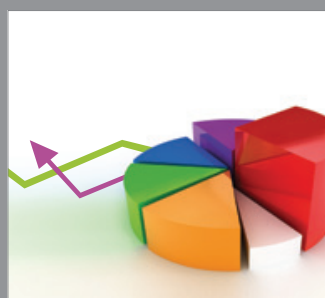

ournal of

Probability and Statistics

Promensencen
